\title{
Inversão Linearizada 2D de Dados Gravimétricos: Critérios de Avaliação dos Resultados da Inversão
}

\author{
Silvia Lorena Bejarano* e Amin Bassrei ${ }^{1}$, CPGG/IGEO/Universidade Federal da Bahia \& INCT/GP
}

Copyright 2016, SBGf - Sociedade Brasileira de Geofísica

Este texto foi preparado para a apresentação no VII Simpósio Brasileiro de Geofísica, Ouro Preto, 25 a 27 de outubro de 2016. Seu conteúdo foi revisado pelo Comitê Ouro Preto, 25 a 27 de outubro de 2016. Seu conteúdo foi revisado pelo Comite
Técnico do VII SimBGt, mas não necessariamente representa a opinião da SBGf ou de seus associados. É proibida a reprodução total ou parcial deste material para propósitos comerciais sem prévia autorização da SBGf.

\begin{abstract}
This work investigates two methodologies for the appraisal of the solutions in the inversion of $2 \mathrm{D}$ gravity data. The first is the well-known concept of resolution matrices, and the second is a method proposed by Barbieri, originally for medical imaging. Besides the numerical computation and the aid of curves, these two estimators can be displayed as images. The simulations using linearized inversion were performed in a synthetic model inspired on a real situation from the Recôncavo Basin, State of Bahia, Brazil. Then, it was used real data from the same basin. In order to attenuate the illposedness of the inverse problem, it was regularization by derivative matrices. The Barbieri method showed to be more effective that the resolution matrices, in such a way that it was possible to evaluate the quality of a given solution or the portions of the solution closer to the true model.
\end{abstract}

\section{Introdução}

O conhecimento do interior da Terra, especialmente seus os parâmetros físicos e geométricos, tem por base o processamento de valores observados na superfície de alguma grandeza física. Trata-se de um complexo processo, chamado de problema inverso, retroalimentado em que o modelo estimado (os parâmetros físicos) pelo processamento é confrontado com os aspectos físicos reais e geológicos. Nesse processo, as informações prévias terminam por impor possíveis estruturas.

A Geofísica dedica seus esforços para obter a solução do problema inverso, mas, em geral, pouca atenção é dada à crítica e ao escrutínio da solução obtida. A validação dos resultados da inversão invertidos é realizada com a interpretação com base na realidade geológica pretendida, o que também é importante. Entre alguns critérios utilizados na prática, tem-se o grau de ajuste ou o erro, entre o dado calculado e o dado observado. Porém, o erro do dado em si não traz informações sobre a qualidade do modelo estimado pela inversão. O objetivo principal deste trabalho é apresentar diferentes metodologias para a avaliação da qualidade da inversão linearizada em dados 2D em gravimetria.
Os problemas inversos são, em geral, mal postos, ou seja, sua solução pode não existir, pode não ser única ou pode não ser estável. Mesmo que a solução exista e seja única, ela pode ser instável, ou seja, uma pequena perturbação nos parâmetros dos dados observados pode causar uma grande perturbação nos parâmetros dos modelos a serem estimados.

Uma boa medida da não-unicidade num problema inverso pode ser obtida através do uso de matrizes de resolução (Jackson, 1972).

Um segundo critério é baseado em Barbieri (1974), que apresenta uma alternativa para a avaliação do processo de inversão. Nesse método, além do vetor solução do problema inverso $\mathbf{m}^{\text {est }}$, existe um segundo vetor $\mathbf{m}^{\text {est,c }}$ denominado estimado complementar que, somado ao primeiro, forma um terceiro vetor, w, cujos elementos, idealmente, seriam idênticos a uma constante préestabelecida. Dessa forma, além da análise dos desvios dos elementos de w em relação ao valor constante, uma outra característica interessantes é o fato que a imagem ou gráfico do vetor $\mathbf{w}$ irá indicar as regiões do modelo em a inversão foi bem sucedida.

Para a modelagem gravimétrica seguimos o trabalho de Murthy e Rao (1992), que utiliza prismas retangulares justapostos lado a lado, representando um corpo geológico 2D. O parâmetro de modelo da inversão é a profundidade da base de cada prisma retangular.

Para avaliar a aplicabilidade desses critérios, foram utilizados modelos sintéticos 2D para simular os dados observados (anomalia de campo gravimétrico), com a inclusão de ruídos de diferentes intensidades. Após isso, os dados foram invertidos com o auxílio de regularização. O conjunto de dados sintéticos utilizado foi inspirado numa situação real da Bacia do Recôncavo. Finalmente, foi utilizado um conjunto de dados reais provenientes da mesma Bacia. Os resultados puderam comprovar a aplicabilidade dos métodos mencionados, de modo que foi possível criticar a qualidade das soluções obtidas.

\section{Matrizes de Resolução}

Segundo Backus e Gilbert (1970), em problemas inversos lineares em Geofísica, a solução deve fazer uso de técnicas de otimização para a obtenção do modelo estimado a partir do dado observado. Existem duas matrizes de resolução. A matriz de resolução do modelo é a matriz que caracteriza a relação entre os parâmetros

\footnotetext{
${ }^{1}$ Atualmente como professor visitante na Universidade de Stanford.
} 
do modelo estimados e verdadeiros (Menke, 1989). A matriz de resolução de modelo mede o quanto o modelo estimado $\mathbf{m}^{\text {est }}$ se aproxima do verdadeiro $\mathbf{m}^{\text {ver }}$, conforme o processo inverso (Jackson, 1972; Menke, 1989; Susan, 1996):

$$
\mathbf{m}^{\text {est }}=\mathbf{G}^{+} \mathbf{d}^{\text {obs }}=\mathbf{G}^{+} \mathbf{G} \mathbf{m}^{\text {ver }} \equiv \mathbf{R}_{\mathrm{m}} \mathbf{m}^{\text {ver }},
$$

onde $\mathbf{R}_{\mathrm{m}}$ é a matriz de resolução do modelo. Se a matriz de resolução for igual à matriz identidade, ou seja, $\mathbf{R}_{\mathrm{m}}=\mathbf{I}$, então $\mathbf{m}^{\text {est }}=\mathbf{m}^{\text {ver }}$. Nessa condição os parâmetros do modelo estão perfeitamente resolvidos na inversão.

A equação (1) incorpora a suposição básica ao método de inversão que define a solução $\mathbf{m}^{\text {est }}$ como uma projeção linear de $\mathbf{m}^{\mathrm{ver}}$ (Backus e Gilbert, 1970). Também, a solução $\mathbf{m}^{\text {est }}$ pode ser vista como a média calculada pela integração sobre o modelo $\mathbf{m}^{\mathrm{ver}}$.

Segundo Wiggins (1972), a principal utilidade da matriz de resolução é fornecer uma medida da resolução obtida a partir dos dados e esta medida é baseada no grau em que a matriz de resolução se aproxima da matriz identidade.

Por seu turno, a matriz de resolução de dado é a matriz que caracteriza a relação entre os dados observados e os dados calculados com um determinado modelo (Menke, 1989). Esta matriz descreve até que ponto as previsões correspondem aos dados. O problema direto representa-se pela equação $\mathbf{d}=\mathbf{G m}$, por sua vez a equação inversa pode ser expressa como:

$$
\mathbf{m}^{\text {est }}=\mathbf{G}^{+} \mathbf{d}^{\text {obs }},
$$

que combinada com a equação

$$
\mathbf{d}^{\mathrm{cal}}=\mathbf{G m}^{\mathrm{est}},
$$

resulta em:

$$
\mathbf{d}^{\text {cal }}=\mathbf{G}\left(\mathbf{G}^{+} \mathbf{d}^{\text {obs }}\right)=\left(\mathbf{G G}^{+}\right) \mathbf{d}^{\text {obs }} \equiv \mathbf{R}_{\mathrm{d}} \mathbf{d}^{\text {obs }},
$$

sendo $\mathbf{R}_{d}$ a matriz de resolução de dado. Esta matriz descreve até que ponto as previsões correspondem aos dados. A matriz de resolução de dado é determinada pelas funções fundamentais do fenômeno físico envolvido e alguma informação a priori adicionada ao problema (Xia et al., 2008) e é independente dos valores presentes nos dados (Menke, 1989).

Para realizar um estudo quantitativo do problema inverso, foram computados erros percentuais da diagonal principal da matriz de resolução de dados $\varepsilon_{\mathrm{Rd}}$, assim como os erros percentuais da diagonal principal da matriz de resolução de modelo $\varepsilon_{\mathrm{Rm}}$. Tais estimadores são expressos, respectivamente, por:

$$
\varepsilon_{\mathrm{R}_{\mathrm{d}}}(\%)=\frac{1}{\mathrm{M}} \sqrt{\sum_{\mathrm{j}=1}^{\mathrm{M}}\left(1-\mathrm{R}_{\mathrm{d}, \mathrm{j}}\right)^{2}} \times 100 \%,
$$

e

$$
\varepsilon_{\mathrm{R}_{\mathrm{m}}}(\%)=\frac{1}{\mathrm{~N}} \sqrt{\sum_{\mathrm{i}=1}^{\mathrm{N}}\left(1-\mathrm{R}_{\mathrm{m}, \mathrm{ii}}\right)^{2}} \times 100 \%
$$

\section{Método de Barbieri}

Outro critério para avaliar o processo de inversão foi sugerido por Barbieri (1974), originariamente aplicado em imageamento médico. Estendendo a abordagem de Barbieri para a Geofísica, a imagem no caso será equivalente à matriz com as propriedades do meio em subsuperfície (parâmetros de modelo). Uma segunda imagem, denominada complementar, similar ao negativo de uma chapa fotográfica, será perfeita se, somada à primeira, resultar numa terceira imagem, ou terceiro vetor w, cujos elementos devem o mesmo valor. Essa imagem (ou vetor) com todos os elementos constantes será denominada de pseudoimagem.

Com base no acima descrito é possível construir a equação (Bassrei, 2000):

$$
\mathbf{m}^{\text {ver }}+\mathbf{m}^{\text {ver,c }}=\mathbf{w},
$$

e, aplicando-se o operador $\mathbf{G}$ à esquerda tem-se:

$$
\mathbf{G m} \mathbf{v e r}^{\text {v }} \mathbf{G} \mathbf{m}^{\text {ver,c }}=\mathbf{G w} \text {. }
$$

Lembrando que $\mathbf{G m}^{\text {ver }}=\mathbf{d}^{\text {obs }}$ e considerando ainda $\mathrm{Gm}^{\mathrm{ver}, \mathrm{c}}=\mathbf{d}^{\mathrm{obs}, \mathrm{c}}$, o vetor complementar dos parâmetros de dado observados $\mathbf{d}^{\text {obs,c }}$ pode ser isolado como:

$$
\mathbf{d}^{\text {obs,c }}=\mathbf{G w}-\mathbf{d}^{\text {obs }} .
$$

Realizando-se uma segunda inversão $\operatorname{com} \mathbf{d}^{\mathrm{obs}, \mathrm{c}}$, se obtém $\mathbf{m}^{\text {est,c }}$, ou seja, o vetor complementar dos parâmetros do modelo estimado. Somado este último vetor ao vetor modelo estimado, se obtém uma pseudoimagem estimada:

$$
\mathbf{w}^{\text {est }}=\mathbf{m}^{\text {est }}+\mathbf{m}^{\text {est,c }} .
$$

Esse resultado será igual à pseudoimagem $\mathbf{w}$ se a inversão tiver sido exata. Como geralmente isso não ocorre, pode-se verificar a qualidade da inversão ao longo da região de estudo, qualitativamente pelo exame visual da pseudoimagem $\mathbf{w}^{\text {est }}$. Portanto, pode se ter uma clara visão se o método de inversão utilizado foi efetivo ou não, seja no modelo como um todo ou em partes do mesmo (Bassrei, 2000).

No problema de inversão com a inclusão de regularização, a equação (7) é multiplicada pelo operador $\mathbf{G}^{\top} \mathbf{G}$ à esquerda de modo que:

$$
\mathbf{G}^{\mathrm{T}} \mathbf{G} \mathbf{m}^{\text {ver }}+\mathbf{G}^{\mathrm{T}} \mathbf{G} \mathbf{m}^{\text {ver,c }=} \mathbf{G}^{\mathrm{T}} \mathbf{G} \mathbf{w},
$$

ou

$$
\mathbf{G}^{\mathrm{T}} \mathbf{d}^{\mathrm{obs}}+\mathbf{G}^{\mathrm{T}} \mathbf{d}^{\mathrm{obs}, \mathrm{c}=} \mathbf{G}^{\mathrm{T}} \mathbf{G} \mathbf{w}
$$

ou ainda

$$
\mathbf{G}^{\mathrm{T}} \mathbf{d}^{\mathrm{obs}, \mathrm{c}=} \mathbf{G}^{\mathrm{T}} \mathbf{G} \mathbf{w}-\mathbf{G}^{\mathrm{T}} \mathbf{d}^{\mathrm{obs}} .
$$

Deste modo, fazendo-se uma segunda inversão com $\mathbf{d}^{\text {obs,c }}$, tem-se:

$$
\mathbf{m}^{\mathrm{est}, \mathrm{c}}=\left(\mathbf{G}^{\mathrm{T}} \mathbf{G}+\lambda \mathbf{D}_{\mathrm{n}}^{\mathrm{T}} \mathbf{D}_{\mathrm{n}}\right)^{+} \mathbf{G}^{\mathrm{T}} \mathbf{d}^{\mathrm{obs}, \mathrm{c}} \text {. }
$$

Novamente, pode se obter $\mathbf{w}^{\text {est }}$ como a soma de $\mathbf{m}^{\text {est }}+$ $\mathbf{m}^{\text {est,c }}$, desta vez com a inclusão de regularização. 
A eficácia da abordagem de Barbieri pode ser determinada de forma quantitativa. $E$ para tanto utilizamos neste trabalho o seguinte estimador:

$$
\varepsilon_{\mathrm{w}}(\%)=\frac{\sqrt{\sum_{\mathrm{i}=1}^{\mathrm{N}}\left(\mathrm{w}_{\mathrm{i}}-\mathrm{w}_{\mathrm{i}}^{\mathrm{est}}\right)^{2}}}{\sqrt{\sum_{\mathrm{i}=}^{\mathrm{N}}\left(\mathrm{w}_{\mathrm{i}}\right)^{2}}} \times 100 \% .
$$

\section{Metodologia}

O propósito destes testes foi avaliar a qualidade da solução do problema inverso, utilizando o critério da matriz de resolução e o método de Barbieri. Os testes foram realizados com a inversão linearizada, utilizando regularização de ordens zero, um e dois. Para cada ordem de regularização foram utilizados diversos valores do parâmetro de regularização $\lambda$. A inversão numérica da matriz hessiana $\left(\mathbf{G}^{\mathrm{T}} \mathbf{G}+\lambda \mathbf{D}_{\mathrm{n}}^{\mathrm{T}} \mathbf{D}_{\mathrm{n}}\right)$ foi através do SVD, decomposição por valores singulares.

A inversão da anomalia de campo Bouguer com o propósito de estimar a profundidade da base dos prismas que compõem o corpo causador é um problema não linear e sua solução será obtida de forma linearizada, com regularização.

Foram utilizados dois modelos. O primeiro é um modelo sintético com 110 prismas, inspirado numa situação real da Bacia do Recôncavo, Bahia. O objetivo é a determinação das profundidades que representam o contato do sedimento com o embasamento. Depois, para a mesma finalidade, foi utilizado um perfil gravimétrico real da Bacia do Recôncavo. Esse último foi escolhido para validar os critérios de avaliação da qualidade da inversão numa situação real, onde não se conhece o modelo verdadeiro, ou seja, as profundidades reais do contato entre o sedimento e o embasamento. Em ambos os casos o número de observações foi maior que o número de prismas, ou seja, ambos foram problemas sobredeterminados.

\section{Dados Sintéticos}

Esse modelo foi estabelecido por Soares (2013), e é apresentado na Figura 1, onde se pode ver a geometria do modelo na parte inferior da figura e a sua anomalia de campo correspondente na parte superior. Segundo Soares (2013), esse modelo é inspirado numa situação real de um perfil 2D da Bacia do Recôncavo. No caso, o corpo 2D foi parametrizado por 110 prismas, sendo cada prisma com uma largura constate de $1,08 \mathrm{~km}$. As profundidades dos prismas variaram desde $50 \mathrm{~m}$ até 6 $\mathrm{km}$. O contraste de densidade foi considerado constante e no valor de $-200 \mathrm{~kg} / \mathrm{m}^{3}$ ou $-0.2 \mathrm{~g} / \mathrm{cm}^{3}$.

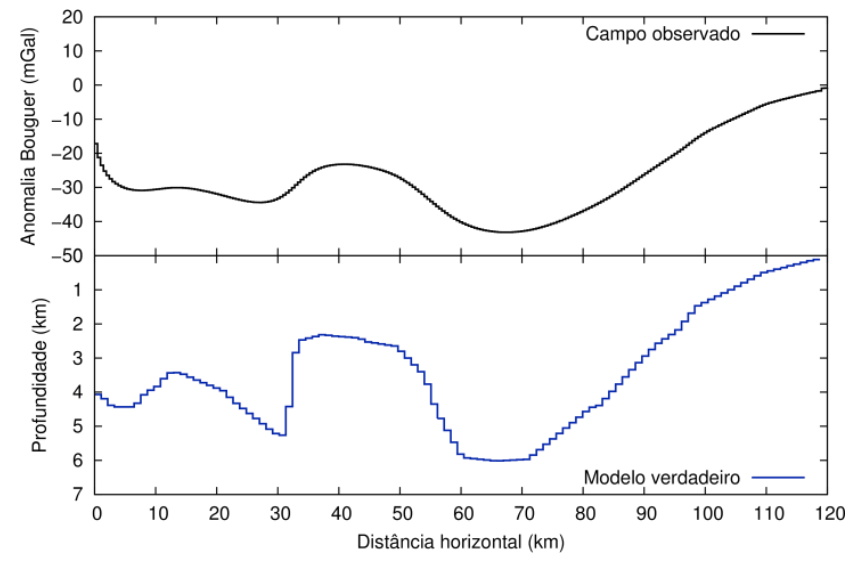

Figura 1: Modelo sintético inspirado num perfil 2D da Bacia do Recôncavo. A geometria do modelo é mostrada na parte inferior da figura e a sua anomalia de campo correspondente na parte superior.

Foram realizadas inversões com regularização de ordens zero, um e dois, assim como foram utilizados os seguintes valores do parâmetro de regularização: $\lambda=10^{-2}$, $10^{-1}, 10^{0}, 10^{1}$ e $10^{2}$.

Para o caso de regularização de ordem 0 , conforme se vê na Tabela 1 , os menores valores foram $\varepsilon_{m}=2.23 \%$ e $\varepsilon_{\mathrm{w}}=0,139 \%$. Para o caso da ordem 1 tivemos $\varepsilon_{\mathrm{m}}=1.84$ $\%$ e $\varepsilon_{w}=0.089 \%$. Por ultimo, para o caso da ordem 2 , o melhor resultado teve $\varepsilon_{m}=1.92 \%$ e $\varepsilon_{w}=0.088 \%$.

Além dos valores do erro RMS relativo entre o modelo verdadeiro e o modelo estimado e o erro RMS relativo entre os dados observados e os dados calculados, a Tabela 1 contém também o estimador expresso pela equação (6) que não se mostrou efetivo para a escolha do parâmetro de regularização e a avaliação da solução do problema inverso. Por outro lado, o estimador expresso pela equação (12) se mostro efetivo. Analisando a Tabela 1 podemos verificar que existe uma correlação entre o erro RMS entre o modelo verdadeiro e recuperado e o estimador expresso pela equação (12), ou seja, $\varepsilon_{\mathrm{w}}$. Os mínimos entre $\varepsilon_{\mathrm{m}}$ e $\varepsilon_{\mathrm{w}}$ coincidiram para as ordens 0 e 1, e foram próximos no caso da regularização de ordem 2.

Por limitação de espaço não é possível mostrar todos os resultados da Tabela 1. A Figura 2(a) mostra o modelo verdadeiro e os modelos estimados para diferentes valores de $\lambda$. O modelo inicial considerado tinha uma profundidade constante e igual a $6 \mathrm{~km}$ para todos os prismas. Além disso, esse resultado se refere para a ordem de regularização 1 (primeira derivada). 


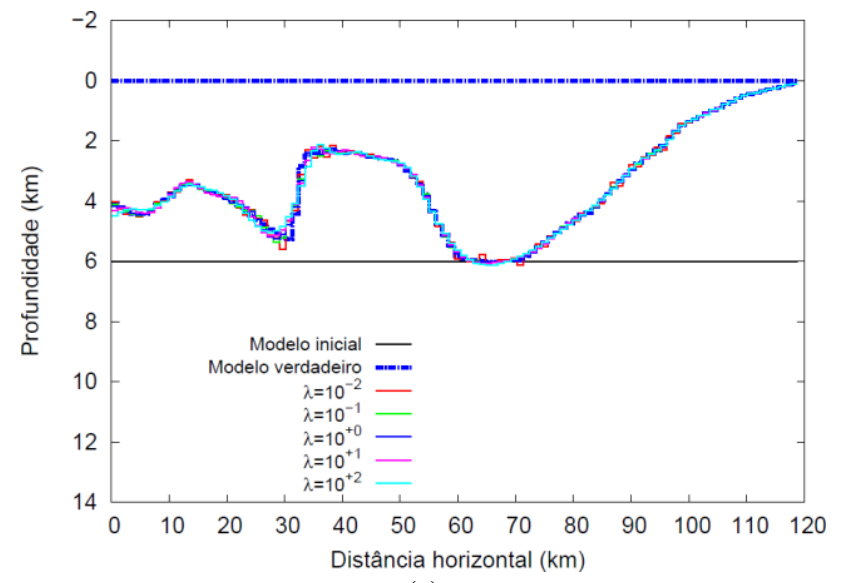

(a)

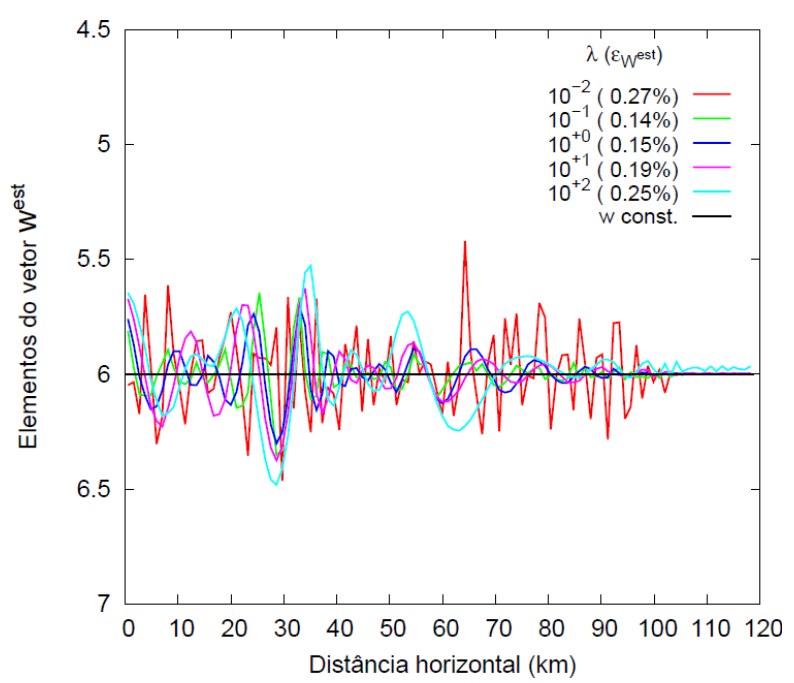

(b)

Figura 2: Resultados da inversão linearizada com regularização de ordem 1, para diferentes parâmetros de regularização $\lambda$. O modelo inicial foi uma profundidade constante e igual a $6 \mathrm{~km}$ para todos os prismas.

A Figura 2(b) representa o que chamamos de pseudoimagens, ou pseudo-curvas no caso $2 \mathrm{D}$, que é o está tratado. Cada ponto do eixo horizontal corresponde à soma da profundidade estimada com a profundidade complementar estimada, conforme apresentado na equação (10). Num caso ideal, em que a inversão fosse exata, esse valor deveria corresponder a $6 \mathrm{~km}$, que foi o valor adotado para os elementos do vetor constante $\mathbf{w}$, conforme a equação (7). Mas o que está plotado é o vetor $\mathbf{w}^{\text {est }}$.

O desvio dos valores da curva em relação ao valor constante de $6 \mathrm{~km}$ corresponde a regiões do modelo onde a inversão não foi bem sucedida. Esse desvio é expresso pelo estimador $\varepsilon_{\mathrm{w}}$. Cada curva da Figura 2(b) está associada a um valor do parâmetro de regularização. $\mathrm{O}$ canto superior da Figura 2(b) informa a correspondência entre cor e parâmetro de regularização, assim como o valor do estimador $\varepsilon_{\mathrm{w}}$, que também listado na última coluna da Tabela 1.

Tabela 1: Resultados da inversão linearizada com regularização de ordens 0,1 e 2 . O modelo inicial foi uma profundidade constante e igual a $6 \mathrm{~km}$ para todos os prismas.

\begin{tabular}{|c|l|l|l|l|l|l|}
\hline$n$ & $\lambda$ & Iterações & \multicolumn{1}{|c|}{$\varepsilon_{\mathrm{d}}(\%)$} & $\varepsilon_{\mathrm{m}}(\%)$ & \multicolumn{1}{|c|}{$\varepsilon_{\mathrm{Rm}}(\%)$} & $\varepsilon_{\mathrm{Rd}}(\%)$ \\
\hline \multirow{5}{*}{0} & $10^{-2}$ & 30 & 0.0106 & 2.95 & 1.33 & 0.268 \\
& $10^{-1}$ & 30 & 0.0194 & 2.23 & 0.119 & 0.139 \\
& $10^{0}$ & 30 & 0.0433 & 2.59 & 0.0133 & 0.146 \\
& $10^{1}$ & 30 & 0.0968 & 3.04 & 0.00171 & 0.192 \\
& $10^{2}$ & 30 & 0.223 & 3.73 & 0.000223 & 0.254 \\
\hline \multirow{6}{*}{1} & $10^{-2}$ & 30 & 0.00994 & 2.35 & 0. & 0.167 \\
& $10^{-1}$ & 30 & 0.0139 & 1.84 & 0.0666 & 0.089 \\
& $10^{0}$ & 30 & 0.0342 & 2.3 & 0.00906 & 0.097 \\
& $10^{1}$ & 30 & 0.0686 & 2.71 & 0.00109 & 0.127 \\
& $10^{2}$ & 30 & 0.128 & 3.15 & 0.000176 & 0.179 \\
\hline \multirow{5}{*}{2} & $10^{-2}$ & 30 & 0.00907 & 2.13 & 0.427 & 0.142 \\
& $10^{-}$ & 30 & 0.0162 & 1.92 & 0.0546 & 0.088 \\
& $10^{0}$ & 30 & 0.0308 & 2.22 & 0.00933 & 0.085 \\
& $10^{1}$ & 30 & 0.0562 & 2.62 & 0.00169 & 0.099 \\
& $10^{2}$ & 30 & 0.104 & 3.02 & 0.000364 & 0.158 \\
\hline
\end{tabular}

Nota-se que o menor erro, ou seja, o melhor valor do parâmetro de regularização foi $\lambda=10^{-1}$ (cor verde) e maior erro, ou seja, o pior valor do parâmetro de regularização foi $\lambda=10^{-2}$ (cor vermelha). O valor $\lambda=10^{-2}$ resultou num comportamento oscilatório mais intenso.

$\mathrm{Na}$ verdade, todos os cinco valores do parâmetro de regularização proporcionaram bons resultados, com erros entre o modelo verdadeiro e o modelo estimado variando entre $1.84 \%$ e $3.15 \%$. Porém, essa metodologia permitiu a escolha do melhor parâmetro de regularização, ou seja, $\lambda=10^{-1}$

Ainda em relação à Figura 2(b) deve-se destacar que a faixa de variação está limitada entre 5.5 e $6.5 \mathrm{~km}$. E, em nenhuma distância horizontal o desvio entre $\mathbf{w}$ e $\mathbf{w}^{\text {est }}$ foi acima de $10 \%$, com exceção para a distância entre 65 e $70 \mathrm{~km}$, e apenas para o parâmetro de regularização $\lambda=$ $10^{-2}$, quando o valor do elemento do vetor $\mathbf{w}^{\text {est }}$ foi inferior a $5.5 \mathrm{~km}$.

No caso do melhor valor do parâmetro de regularização a Figura 2(b) indicou que a inversão foi menos bem sucedida na região horizontal entre 20 a $35 \mathrm{~km}$.

Finalmente, pudemos verificar que a Tabela 1 indicou que o valor do estimador $\varepsilon_{\mathrm{w}}$ diminuiu com o aumento da ordem da regularização. 


\section{Dados Reais}

Com o propósito de validar a metodologia proposta na inversão linearizada com regularização, foram utilizados dados reais da Bacia do Recôncavo, obtidos no trabalho de Soares (2013). As inversões foram realizadas com base no modelo 2D formado por prismas com seções retangulares, lateralmente justapostos, com a mesma profundidade para seus topos e profundidades distintas das bases, sendo estes últimos o conjunto de parâmetros a serem recuperados pela inversão.

Foram utilizadas ordens de regularização zero, um e dois, sendo os valores de regularização $\lambda=10^{-2}, 10^{-1}, 10^{0}$ e $10^{1}$. Foi estabelecido um máximo de trinta iterações e o modelo inicial tinha uma profundidade constante de $3 \mathrm{~km}$, para todos os prismas. O perfil 2D tinha um comprimento de $118,8 \mathrm{~km}$, sendo a largura de cada prisma igual a 1,08 $\mathrm{km}$, de modo que o número de prismas foi 110 . O número de estações em superfície foi 231 , de modo que o problema tratado é do tipo sobredeterminado.

Considerando os parâmetros adotados, os resultados das inversões realizadas com a anomalia de campo observado dessa linha, representado na Figura 3, estão apresentados na Figura 4(a) a 4(f), na forma de modelos estimados e também as respectivas pseudoimagens estimadas. Todos os resultados das simulações estão

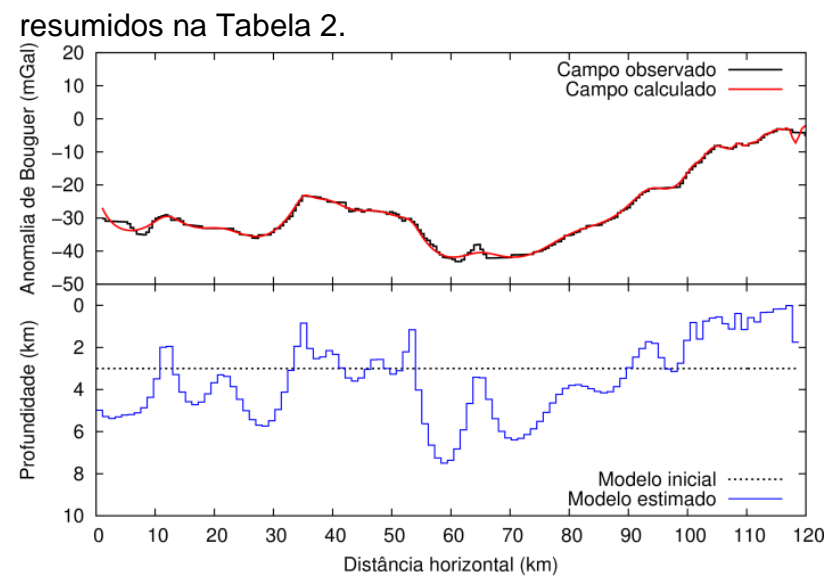

Figura 3: Dados observados e calculados de um perfil gravimétrico na Bacia do Recôncavo na parte superior. Modelo estimado (profundidade em $\mathrm{km}$ ) a partir da inversão linearizada com regularização de ordem zero e parâmetro de regularização $\lambda=10$ na parte inferior.

O modelo selecionado está na Figura 3, juntamente com os perfis gravimétricos observados e calculados. Pode-se afirmar que o método de Barbieri permitiu decidir sobre o melhor resultado das inversões. O resultado com 0 menor desvio de $\varepsilon_{\mathrm{w}}$. Foi relacionado ao parâmetro $\lambda=10$.

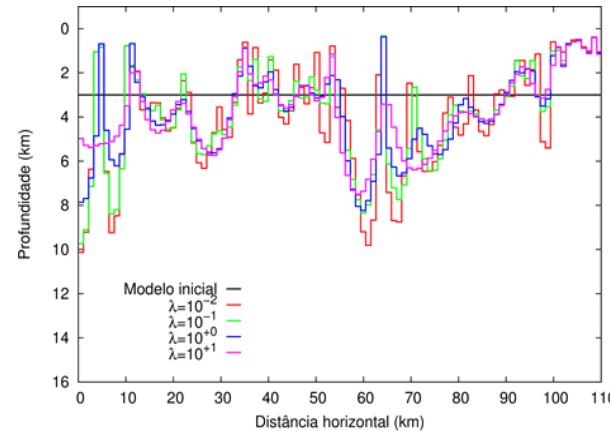

(a)

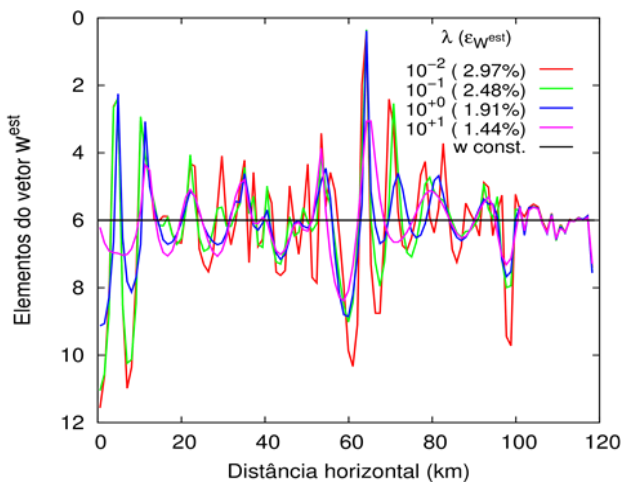

(d)

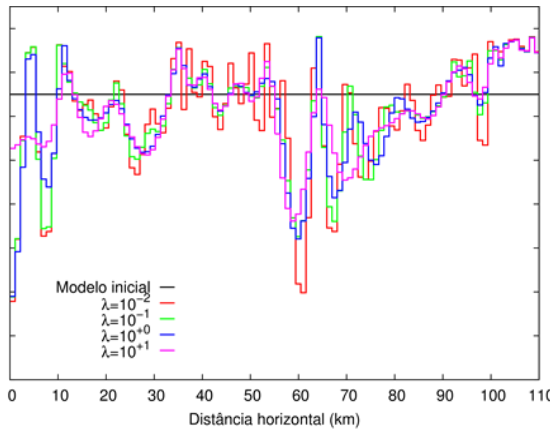

(b)

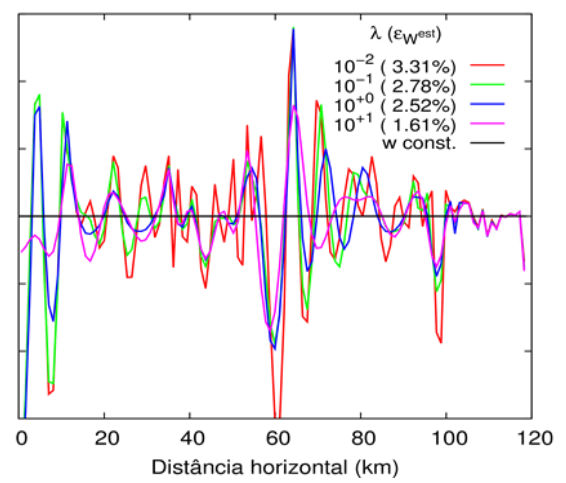

(e)

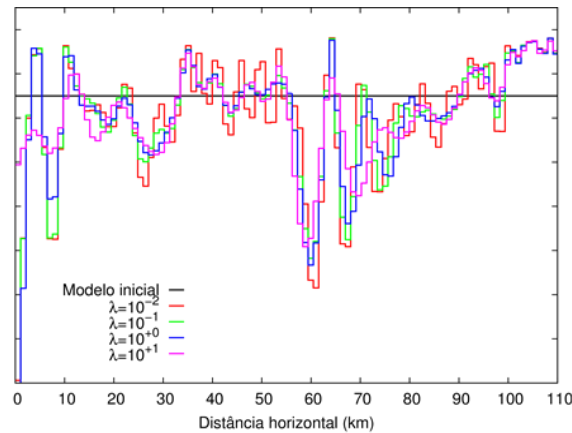

(c)

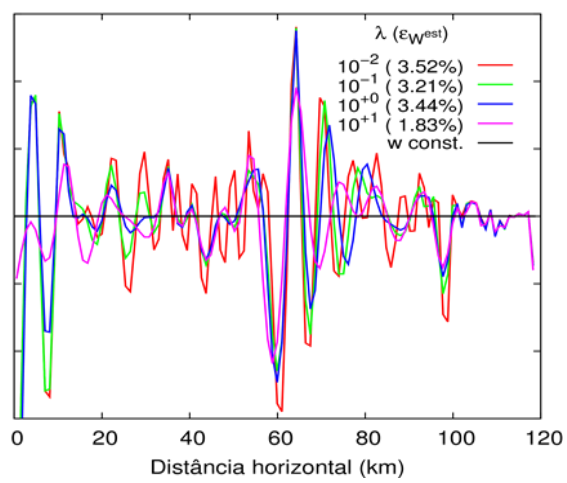

(f)

Figura 4: Inversões linearizadas com diferentes parâmetros de regularização $\lambda$, do perfil gravimétrico da Bacia do Recôncavo com regularização de ordens 0,1 e 2, respectivamente em (a), (b) e (c). As pseudo-imagens associadas estão apresentadas, respectivamente, em (d), (e) e (f). 
Tabela 2: Resultados das inversões linearizadas com regularização dos dados reais da Bacia do Recôncavo.

\begin{tabular}{|c|l|c|l|l|}
\hline$n$ & $\lambda$ & Iterações & $\varepsilon_{\mathrm{d}}(\%)$ & \multicolumn{1}{|c|}{$\varepsilon_{\mathrm{w}}(\%)$} \\
\hline \multirow{3}{*}{0} & $10^{-2}$ & 30 & 1.8 & 2.97 \\
& $10^{-1}$ & 30 & 1.85 & 2.48 \\
& $10^{0}$ & 30 & 2.07 & 1.91 \\
& $10^{1}$ & 30 & 2.71 & 1.44 \\
\hline \multirow{4}{*}{1} & $10^{-2}$ & 30 & 1.78 & 3.31 \\
& $10^{-1}$ & 30 & 1.86 & 2.78 \\
& $10^{0}$ & 30 & 1.99 & 2.52 \\
& $10^{1}$ & 30 & 2.64 & 1.61 \\
\hline \multirow{3}{*}{2} & $10^{-2}$ & 30 & 1.79 & 3.52 \\
& $10^{-1}$ & 30 & 1.85 & 3.21 \\
& $10^{0}$ & 30 & 1.93 & 3.44 \\
& $10^{1}$ & 30 & 2.58 & 1.83 \\
\hline
\end{tabular}

\section{Discussão e Conclusões}

O principal foco deste trabalho foi estudar dois critérios de avaliação do desempenho de inversões linearizadas, as matrizes de resolução e o critério de Barbieri (1974). Os resultados obtidos na inversão 2D de dados gravimétricos permitiram estabelecer as seguintes conclusões.

No caso do modelo sintético verificou uma grande mudança nos comprimentos dos prismas ao longo do perfil, apresentando regiões de menor profundidade também na parte central, cercado por trechos com rápida variação, podendo representar falhas geológicas.

Considerando os resultados obtidos com diversos modelos iniciais e com diversas ordens de regularização, pode-se concluir que o método Barbieri é efetivo para se decidir a respeito da qualidade da inversão, especialmente sobre o modelo estimado a escolher, entre os diversos resultados que se obtém variando-se os parâmetros e a ordem da regularização.

Para menores valores do parâmetro $\lambda$, os modelos estimados apresentam-se por vezes com fortes variações que, provavelmente, não condizem com a realidade geológica. Porém, considerando o que foi visto quando se tratou da inversão com dados sintéticos, é importante acompanhar como se comportam os elementos do vetor $\mathbf{w}^{\text {est }}$, pois dois aspectos se destacam: (i) os valores máximos e mínimos indicam, no caso, que o valor da profundidade obtida para o prisma naquela posição tem, provavelmente, desvios em relação ao verdadeiro, e (ii) as oscilações ao longo do perfil refletem o mesmo comportamento no modelo estimado, indicando um maior ou menor grau de confiabilidade do resultado da inversão. Verifica-se, então, que o método Barbieri é mais efetivo para discriminar essas situações, contando com a ajuda do estimador $\varepsilon_{w}$.

\section{Agradecimentos}

Silvia Bejarano agradece ao $\mathrm{CNPq}$ pela bolsa de doutorado e pela bolsa DTI. A. Bassrei agradece ao $\mathrm{CNPq}$ e à Universidade de Stanford pelo apoio à sua estada de um ano como professor visitante. Ambos os autores agradecem ao CNPq e à PETROBRAS pelo apoio ao projeto INCT-GP e à FINEP pelo apoio ao projeto Rede 01 . Os dados de gravimetria provenientes da ANP foram adquiridos pelo CPGG/UFBA.

\section{Referências}

Backus, G., e Gilbert, F., 1970. Uniqueness in the inversion of inaccurate gross Earth data. Philosophical Transactions of the Royal Society A, vol. 266: 123-192.

Barbieri, M., 1974. A criterion to evaluate three dimensional reconstructions from projections of unknown structures. Journal to Theoretical Biology, vol. 48: 451467.

Bassrei, A., 2000. Novel approaches for the solution and solution evaluation of linear and non-linear inverse problems in geophysics. Boletim da Sociedade Brasileira de Matemática Aplicada e Computacional, vol.4: 1-7.

Jackson, D. D., 1972. Interpretation of inaccurate, insufficient and inconsistent data. Geophysical Journal of the Royal Astronomical Society, vol. 28: 97-109.

Menke, W., 1989. Geophysical Data Analysis: Discrete Inverse Theory: Academic Press.

Murthy, I. V. R., e Rao, S. J., 1992. Gravity inversion of closed two-dimensional bodies. Bollettino di Geofisica Teorica ed Applicata, vol. 34:287-296.

Soares, D., 2013. Inversão de dados gravimétricos 2-D pelos métodos de Levenberg-Marquardt, resfriamento simulado muito rápido e híbrido. Dissertação de Mestrado, Universidade Federal da Bahia, Salvador, BA, Brasil.

Susan, E. M., 1996. A computationally feasible approximate resolution matrix for seismic inverse problems. Geophysical Journal International, vol. 26: 345359.

Wiggins, R. A., 1972. The general linear inverse problem: implication of surface waves and free oscillations for Earth structure. Reviews of Geophysics and Space Physics, vol. 10: 251-285.

Xia, J., Miller, R. e Xu, Y., 2008. Data-resolution matrix and model-resolution matrix for Rayleigh-wave inversion using a damped least-squares method. Pure and Applied Geophysics, vol. 165: 1227-1248. 